\title{
Dosimetric characterization of a synthetic single crystal diamond detector in clinical radiation therapy small photon beams
}

\author{
I. Ciancaglioni, Marco Marinelli, E. Milani, G. Prestopino, C. Verona, and G. Verona-Rinatia) \\ INFN-Dipartimento di Ingegneria Industriale, Università di Roma "Tor Vergata",Via del Politecnico 1, \\ I-00133 Roma, Italy \\ R. Consorti and A. Petrucci \\ U.O. Fisica Sanitaria, Ospedale San Filippo Neri, Via G. Martinotti 20, 00135 Roma, Italy \\ F. De Notaristefani \\ INFN-Dipartimento di Fisica, Università Roma 3, Via della Vasca Navale 84, 00146 Roma, Italy
}

(Received 9 February 2012; revised 30 May 2012; accepted for publication 31 May 2012; published 2 July 2012)

\begin{abstract}
Purpose: To determine the potentialities of synthetic single crystal diamond Schottky diodes for accurate dose measurements in radiation therapy small photon beams.

Methods: The dosimetric properties of a diamond-based detector were assessed by comparison with a reference microionization chamber. The diamond device was operated at zero bias voltage under irradiation with high-energy radiotherapic photon beams. The stability of the detector response and its dose and dose rate dependence were measured. Different square field sizes ranging from $1 \times 1 \mathrm{~cm}^{2}$ to $10 \times 10 \mathrm{~cm}^{2}$ were used during comparative dose distribution measurements by means of percentage depth dose curves (PDDs), lateral beam profiles, and output factors. The angular and temperature dependence of the diamond detector response were also studied.

Results: The detector response shows a deviation from linearity of less than $\pm 0.5 \%$ in the $0.01-7$ Gy range and dose rate dependence below $\pm 0.5 \%$ in the $1-6 \mathrm{~Gy} / \mathrm{min}$ range. PDDs and output factors are in good agreement with those measured by the reference ionization chamber within $1 \%$. No angular dependence is observed by rotating the detector along its axis, while $\sim 3.5 \%$ maximum difference is measured by varying the radiation incidence angle in the polar direction. The temperature dependence was investigated as well and a $\pm 0.2 \%$ variation of the detector response is found in the $18-40{ }^{\circ} \mathrm{C}$ range.

Conclusions: The obtained results indicate the investigated synthetic diamond-based detector as a candidate for small field clinical radiation dosimetry in advanced radiation therapy techniques. (C) 2012 American Association of Physicists in Medicine. [http://dx.doi.org/10.1118/1.4729739]
\end{abstract}

Key words: narrow field dosimetry, synthetic diamond detector, clinical dosimetry, microionization chamber

\section{INTRODUCTION}

The introduction of advanced radiation therapy techniques involving the superimposition of narrow high-gradient photon beams, such as intensity modulated radiation therapy (IMRT) and stereotactic radiosurgery (SRS) has produced an increasing interest in high-resolution dosimetry. In particular, the need of novel dosimetric techniques has become even more evident due to the availability of innovative technology such as CyberKnife, Gamma Knife, and Tomotherapy.

Due to dose averaging effects and to fluence perturbations caused by their large dimensions, most standard detectors designed for large fields are unsuitable to accurately measure doses in small fields $\left(<3 \times 3 \mathrm{~cm}^{2}\right)$, characterized by highdose gradients and lack of charged particle equilibrium. ${ }^{1-4}$ The development of high-resolution small volume detectors, as microionization chambers and solid state detectors, was studied by several authors with the aim of determining new devices and methods for accurate dosimetry in high-energy narrow photon beams. ${ }^{5-8}$ However, no device was found yet, matching all the features requested to an "ideal" detector for small field dosimetry. PTW PinPoint $0.015 \mathrm{~cm}^{3}$ microionization chambers (PTW, Freiburg, Germany), as types 31006 (no longer available) and 31014 , were extensively examined ${ }^{8-11}$ and assessed as proper dosimeters in the field size range 2 $\times 2 \mathrm{~cm}^{2}$ to $10 \times 10 \mathrm{~cm}^{2}$. Volume averaging effects due to the finite dimension of the device sensitive area, radiation induced leakage currents, and polarity effects are the main causes of under-response to smaller fields and over-response to the larger ones. ${ }^{9,10,12}$ Better dosimetric performance was obtained from solid-state detectors ${ }^{6,13-15}$ and liquid filled ion chambers ${ }^{16,17}$ in terms of spatial resolution in narrow beam penumbra analysis and accurate output factor measurements.

Diamond has long been considered a suitable material for the construction of small volume high-resolution radiation detectors due to its radiation hardness, near tissue-equivalence, small size, high-sensitivity, and low leakage current. ${ }^{18-24}$ Most papers on diamond dosimeters are dedicated to commercial natural diamond-based detectors from PTW. Polycrystalline chemical vapor deposition (CVD) diamond ${ }^{25-28}$ and CVD or high-pressure high-temperature (HPHT) 
synthetic single crystal diamond ${ }^{29-31}$ have been also studied as radiation therapy dosimeters. However, lack of reproducibility, difficulties in controlling impurities incorporation in synthetic crystals, and problems related to encapsulation techniques make the performance of such devices still far from the ones of PTW natural diamond detectors.

Recently, synthetic single crystal diamond detectors (SCDDs) in a Schottky diode configuration were realized and tested as radiotherapy dosimeters with different beam qualities (photons from ${ }^{60} \mathrm{Co}$ to $10 \mathrm{MV}$ and electrons from $6 \mathrm{MeV}$ to $18 \mathrm{MeV}$ ), for relative dosimetry. ${ }^{32-34}$ In addition, a detailed characterization of SCDDs as absolute dosimeters was performed in the framework of a collaboration with the Italian Metrology Institute (ENEA-INMRI), involving both comparison with primary standards and Monte Carlo simulations. ${ }^{34}$

In the present paper a detailed dosimetric characterization of such device is presented under $10 \mathrm{MV}$ photon beam irradiations. Particular care was devoted to the response in small field sizes, in view of its application in advanced radiation therapy techniques.

Even though a single diamond dosimeter was investigated in the present work, several detectors (more than 50) have been fabricated and tested. Similar dosimetric properties were obtained for all the devices. However, a comprehensive and systematic investigation was not performed for all samples.

\section{EXPERIMENTAL}

\section{II.A. Diamond and reference detectors}

The synthetic SCDD studied in the present paper was fabricated at Roma "Tor Vergata" University laboratories by a two-step microwave plasma enhanced CVD. A conductive boron-doped layer, with acceptors concentration of about $5 \times 10^{19} \mathrm{~cm}^{-3}$, was first grown as a back contact on a commercial $4 \times 4 \times 0.4 \mathrm{~mm}^{3}$ HPHT Ib single crystal diamond, on top of which a nominally intrinsic diamond film was deposited. ${ }^{35}$ Finally, a thin circular rectifying aluminum contact about $2.2 \mathrm{~mm}$ in diameter was thermally evaporated on the intrinsic diamond surface, while annealed ohmic silver contacts were formed on the boron-doped layer. The intrinsic diamond layer is therefore the only sensitive volume, no contribution coming from the HPHT substrate. The device acts as a diamond-based Schottky-barrier photodiode, which can be operated both at zero bias voltage (photovoltaic mode) and under reverse bias (photodiode mode), with a positive voltage applied to the $\mathrm{Al}$ contact. ${ }^{35}$ In the present study, the device was always operated in photovoltaic mode.

A $1.0 \pm 0.1 \mu \mathrm{m}$ thick intrinsic diamond layer was used for the detector investigated in this paper, resulting in a sensitive volume of about $0.0038 \mathrm{~mm}^{3}$. The SCDD was embedded in a polymethyl-methacrylate (PMMA) waterproof cylindrical housing, $8 \mathrm{~mm}$ in diameter and $35 \mathrm{~mm}$ long, filled by epoxy resin. The diamond surface is located $1.5 \mathrm{~mm}$ below the top surface of the housing. At the end of the encapsulation process, the cylindrical probe was covered with a conductive graphite-based lacquer used as a shield to reduce the noise from external signals. In Fig. 1(a) schematic, a picture
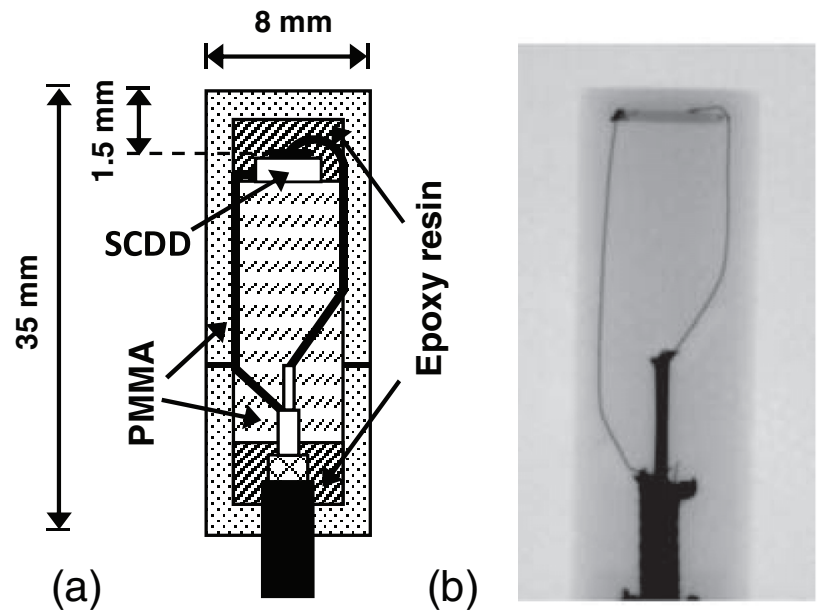

(b)

(c)

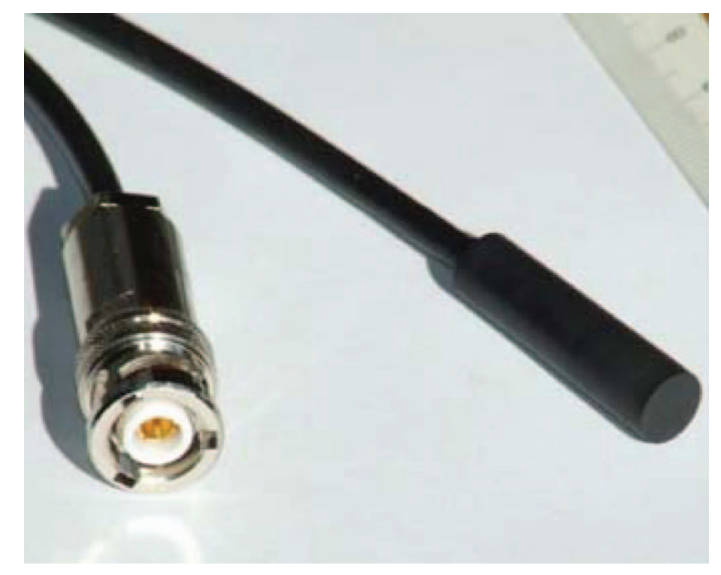

FIG. 1. (a) Longitudinal cross section of the encapsulated diamond detector, (b) x-ray image of the device, and (c) photo of the waterproof housing and of the triaxial connector.

and a radiographic x-ray image of the SCDD are shown. The diamond plate and the electrical connections are clearly visible in the x-ray image and the alignment of the center of the sensitive volume with the geometrical center of the PMMA housing can be appreciated. In the present study the device was tested with its axis parallel to the beam direction (vertical orientation in the following) to minimize possible stem effects, unless otherwise specified. The reference measurement point was then assumed at the center of the top surface of the intrinsic diamond layer, $1.5 \mathrm{~mm}$ below the detector tip. Moreover, the detector alignment with respect to the central axis (CAX) was carefully verified by performing, both in-plane (gun-target) and cross-plane (left-right) profile measurements at various depths for all the field sizes studied. ${ }^{36}$

The dosimetric properties of the SCDD were assessed by comparison with a PTW type 31014 PinPoint $15 \mathrm{~mm}^{3}$ microionization chamber (PP-IC in the following), operated at $400 \mathrm{~V}$ bias voltage, also placed in vertical orientation unless otherwise specified. The nominal cylindrical collecting volume dimensions are $2 \mathrm{~mm}$ diameter by $5 \mathrm{~mm}$ length. The measuring point of the PP-IC for this orientation was assumed $2 \mathrm{~mm}$ below the tip, according to PTW indication. Its position was verified by comparing percentage depth dose (PDDs) 
measured by the PP-IC in vertical and horizontal orientation in fields larger than $3 \times 3 \mathrm{~cm}^{2}$.

\section{II.B. Measurement details}

All measurements reported in this paper were performed under $10 \mathrm{MV}$ beams produced by a Varian Clinac DHX accelerator (Varian Medical Systems, Palo Alto, CA, USA). The square shaped radiation fields, ranging from $1 \times 1 \mathrm{~cm}^{2}$ to $10 \times 10 \mathrm{~cm}^{2}$, were defined by jaws alone, with multileaf collimator fully retracted. Both SCDD and PP-IC were used into a PTW MP3 motorized water phantom with its surface at a distance $\mathrm{SSD}=100 \mathrm{~cm}$ from the source. According to the International Atomic Energy Agency dosimetry protocol ${ }^{37}$ the $10 \times 10 \mathrm{~cm}^{2}$ field size was assumed as a reference field. Absolute dose calibration condition was 1 cGy/MU at the depth of dose maximum $\left(\mathrm{d}_{\max }\right)$ of $2.4 \mathrm{~cm}$ and $\mathrm{SSD}=100 \mathrm{~cm}$.

Temporal behavior and time stability of the diamond-based detector response were studied by connecting the SCDD to a remotely controlled Keithley 6517A picoammeter (Keithley Instruments Inc. Cleveland, Ohio). The SCDD was positioned at $\mathrm{d}_{\max }$ and irradiated with a $10 \times 10 \mathrm{~cm}^{2}$ field at a dose rate of $3 \mathrm{~Gy} \mathrm{~min}{ }^{-1}$, corresponding to $300 \mathrm{MU} \mathrm{min}{ }^{-1}$. A PTW Unidos E Universal Dosimeter was used for the analysis of linearity, dose rate dependence, output factor measurements, angular and temperature dependence.

The dose dependence of the SCDD response was studied at a fixed dose rate of $300 \mathrm{MU} \mathrm{min}{ }^{-1}$, in $2 \times 2 \mathrm{~cm}^{2}$ and $10 \times 10 \mathrm{~cm}^{2}$ field sizes, with the detector positioned at a depth of $10 \mathrm{~cm}$ in the water phantom in the dose range of 2-1000 MU.

The dose rate dependence was investigated in the whole available range at a fixed position, that is, from 100 to $600 \mathrm{MU} \mathrm{min}{ }^{-1}$, by irradiating the device in 6 steps, $200 \mathrm{MU}$ each.

Measurements for output factors determination were also performed at $10 \mathrm{~cm}$, with both SCDD and PP-IC. The OFs were calculated by the ratio between the charge measured for each field size and the reference one.

Depth dose curves and beam profiles were acquired using the PTW MP3 water phantom. For the latter, the SCDD and the PP-IC were positioned at $2.4 \mathrm{~cm}$ and $10 \mathrm{~cm}$ deep in water. An acquisition step of about $0.4 \mathrm{~mm}$ was chosen, corresponding to about $1 / 5$ of the diameter of the detector active region. Cross- and in-plane profiles were recorded by the SCDD and the PP-IC for $1 \times 1,2 \times 2,3 \times 3,4 \times 4,5 \times 5$, and 10 $\times 10 \mathrm{~cm}^{2}$ square fields. The two detectors were used both in vertical and horizontal orientation.

The angular dependence of the diamond detector response was tested with the SCDD placed in the MP3 water phantom at $\mathrm{d}_{\max }$. A $3 \times 3 \mathrm{~cm}^{2}$ field size was used to reduce possible spurious signals from cable irradiation. The angle was varied by rotating the sample by several steps with respect to the beam axis while keeping fixed the position of reference measurement points. ${ }^{6}$ The position of the reference point was carefully verified after each rotation step by using the built in laser alignment system of the Linac and a precision mechan- ical goniometer. In these measurements, two angular dependencies were studied: azimuth angle dependence, with the detector placed in horizontal orientation and rotated around its own longitudinal axis, and polar angle dependence, with the detector long axis tilted with respect to the beam direction. In the latter case the measurement was repeated at a depth of $10 \mathrm{~cm}$.

Finally, the temperature stability was studied between 18 and $40{ }^{\circ} \mathrm{C}$ in a mini water phantom PTW MP1. Since a reduced amount of water was used in order to shorten the time needed to reach thermal equilibrium, the detector was placed at a depth of $5 \mathrm{~cm}$. A K-type thermocouple was used to measure the temperature.

\section{RESULTS AND DISCUSSION}

\section{III.A. Response stability, response time, and sensitivity of the SCDD}

International dosimetry protocols recommend ionization chambers used in radiation therapy to be preirradiated until a stable reading is obtained. ${ }^{37,38}$ Indeed, radiation therapy dosimeters based on semiconductor materials require a preirradiation dose, mainly determined by polarization effects due to filling of deep traps, as well as by radiationinduced conductivity in the insulating materials used for the device encapsulation. ${ }^{27,38-40}$ Results reported in literature for commercial detectors based on natural single crystal diamond indicate a relatively poor intersample reproducibility of the preirradiation dose required to reach a stable response. ${ }^{21-24,41}$ Values ranging from 5 to 15 Gy are reported, depending on the specific impurity concentration into the sample.

In Fig. 2 the current measured by the SCDD at $d_{\max }$ during preirradiation in a $10 \times 10 \mathrm{~cm}^{2}$ field is shown. In spite of the few tens pA detected current, a full range scale of $2 \mathrm{nA}$ was selected for the picoammeter to keep the instrument response time fast enough. After a $10 \mathrm{~min}$ irradiation at a dose rate of $300 \mathrm{MU} \mathrm{min}-1$ the beam was switched on and off three

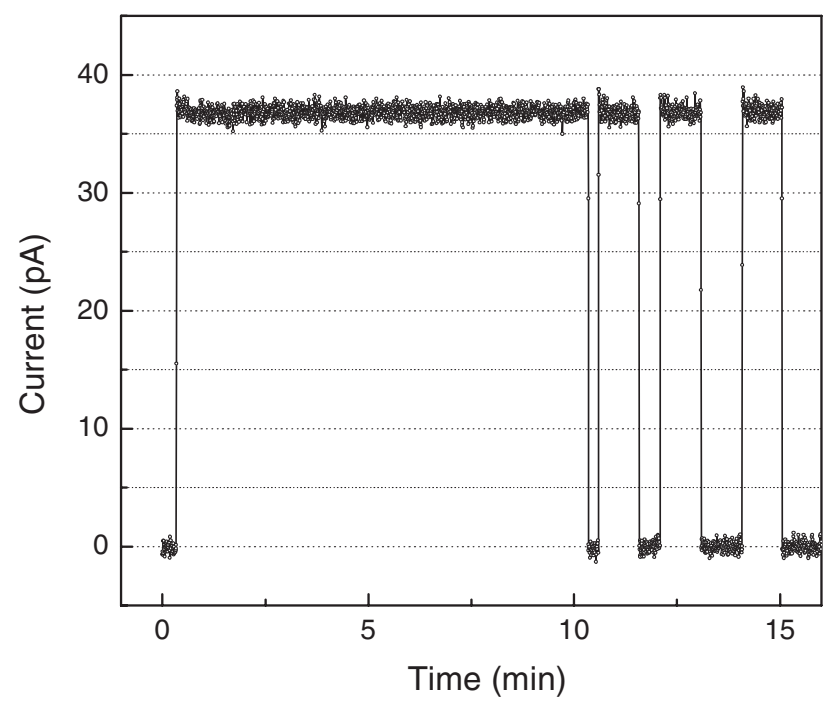

FIG. 2. SCDD response during preirradiation (see text). 
times at the same dose rate, each step lasting $1 \mathrm{~min}$. This was done both to determine the device preirradiation dose needed to achieve a stable signal and to study its response time under irradiation.

From the analysis of the data reported in Fig. 2, a signal stability within $\pm 0.5 \%$ is achieved after $12 \mathrm{~s}$, corresponding to a 0.6 Gy preirradiation dose. Slow response times or memory effects were never observed after the first preirradiation step and a response time faster than $0.1 \mathrm{~s}(10 \mathrm{~Hz}$ acquisition rate) was demonstrated. As for the long term stability of the dosimeter, a variation of the device response below $0.3 \%$ was observed after 2 months by testing it in nominally identical conditions.

A sensitivity of $0.72 \pm 0.02 \mathrm{nC} \mathrm{Gy}^{-1}$ was derived from the value of the SCDD response by comparison with the PP-IC and verified using a PTW 31010 Semiflex calibrated chamber. The uncertainty on the SCDD sensitivity was calculated by combining all the uncertainties coming from both the crosscalibration procedure and the experimental setup, with a coverage factor $\mathrm{k}=2$.

\section{III.B. Dose and dose rate dependence}

The dose dependence of the detector response is reported in Fig. 3(a) for the $10 \times 10 \mathrm{~cm}^{2}$ and $2 \times 2 \mathrm{~cm}^{2}$ field sizes. In both cases the $\mathrm{R}^{2}$ parameter of the linear best fit is found to be 1 with a precision of $10^{-6}$. In Fig. 3(b), the percentage

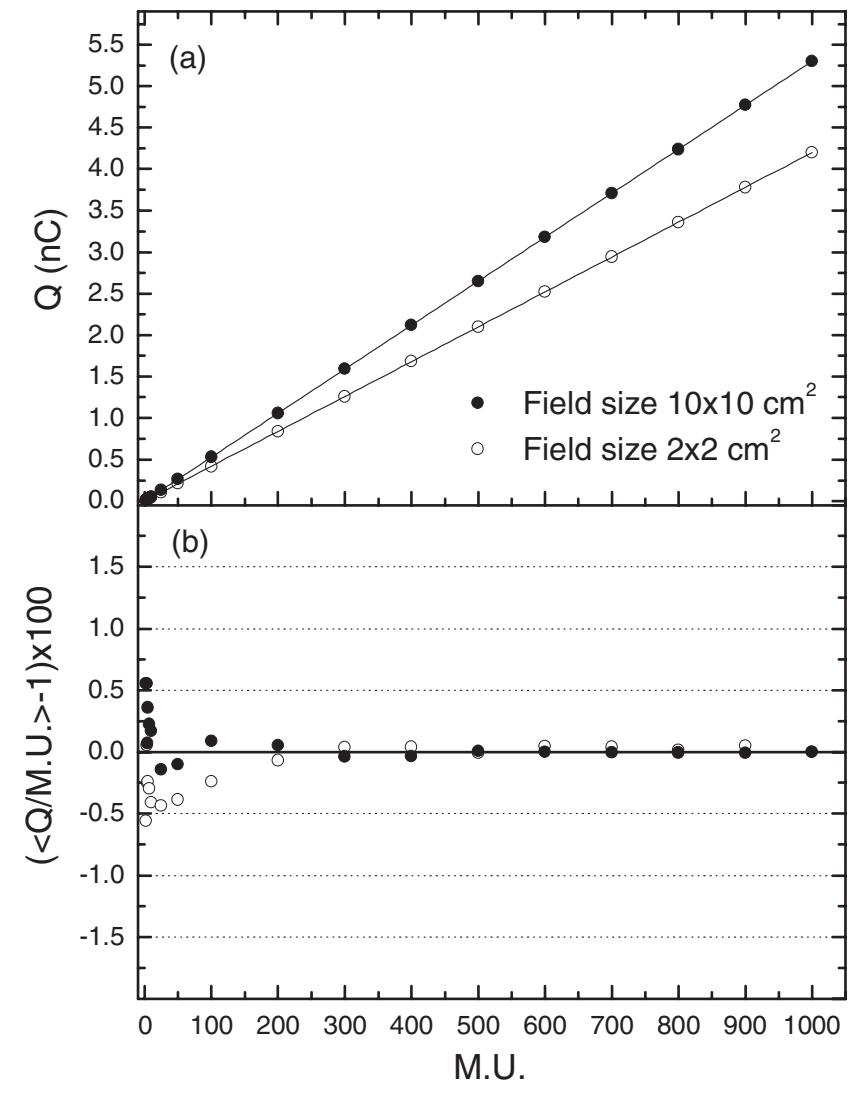

FIG. 3. (a) Measured charge vs delivered MU in $10 \times 10 \mathrm{~cm}^{2}$ and a $2 \times$ $2 \mathrm{~cm}^{2}$ fields at a dose rate of $300 \mathrm{MU} \mathrm{min}-1$ (full lines are linear best fits) and (b) percentage deviations of the measured charge per monitor unit with respect to the one obtained at $1000 \mathrm{MU}$ (b).

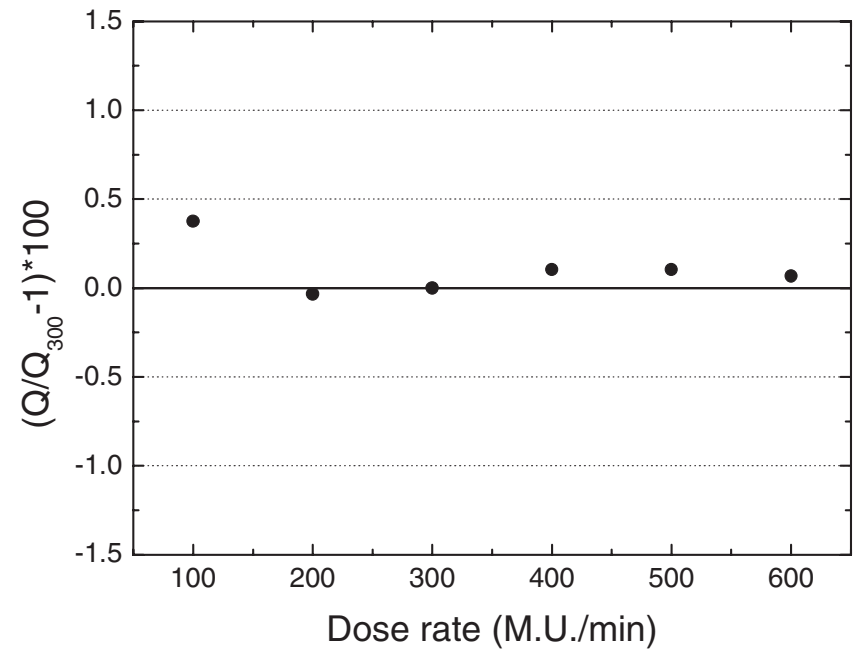

FIG. 4. Percentage deviation of the measured charge with respect to the one measured at $300 \mathrm{MU} / \mathrm{min}$ as a function of the dose rate.

deviations of the measured charge per monitor unit (Q/MU) with respect to the one obtained at $1000 \mathrm{MU}$ is reported. A response linearity within $\pm 0.5 \%$ deviation can be appreciated down to the minimum delivered dose of 2 MU corresponding to about $1.2 \mathrm{cGy}$ in the case of the $2 \times 2 \mathrm{~cm}^{2}$ field. For such a low dose, a signal to noise ratio of about 200 was measured by considering the statistical fluctuation of different measurements. This justifies the data scattering of about $\pm 0.5 \%$ observed in the low dose region of Fig. 3(b).

The dose rate dependence is shown in Fig. 4. In particular, the percentage deviation of the measured charge with respect to the one measured at $300 \mathrm{MU} / \mathrm{min}$ is reported as a function of the dose rate. A deviation below $\pm 0.5 \%$ is observed in the whole investigated dose rate range indicating that no correction factor is necessary for the dosimetric characterization discussed in Secs. III.C-III.F.

\section{III.C. Percentage depth doses and beam profiles}

Central-axis percentage depth dose curves were measured for several square field sizes from $1 \times 1 \mathrm{~cm}^{2}$ to $10 \times 10 \mathrm{~cm}^{2}$. In Fig. 5(a) the PDDs relative to the largest and the smallest utilized field, for both the SCDD and the PP-IC are shown. The relative differences between PDDs measured by the two dosimeters were calculated and reported in Fig. 5(b). Maximum deviations of the order of $1 \%$ can be noticed. Similar behavior is observed for all the investigated field sizes. In particular, the $\mathrm{d}_{\max }$ positions and the PPD values at $10 \mathrm{~cm}$, measured by both SCDD and PP-IC are reported in Table I for all the investigated field sizes. The good agreement between the two devices also suggests that the actual measurement point of the SCDD detector is coincident with the assumed reference point within the positioning error.

In Fig. 6 the in-plane normalized profiles measured at $2.4 \mathrm{~cm}$ with the detectors positioned in vertical orientation are reported (upper curves), together with the differences between the SCDD and the PP-IC profiles calculated for each examined field size (lower curves). A good agreement 


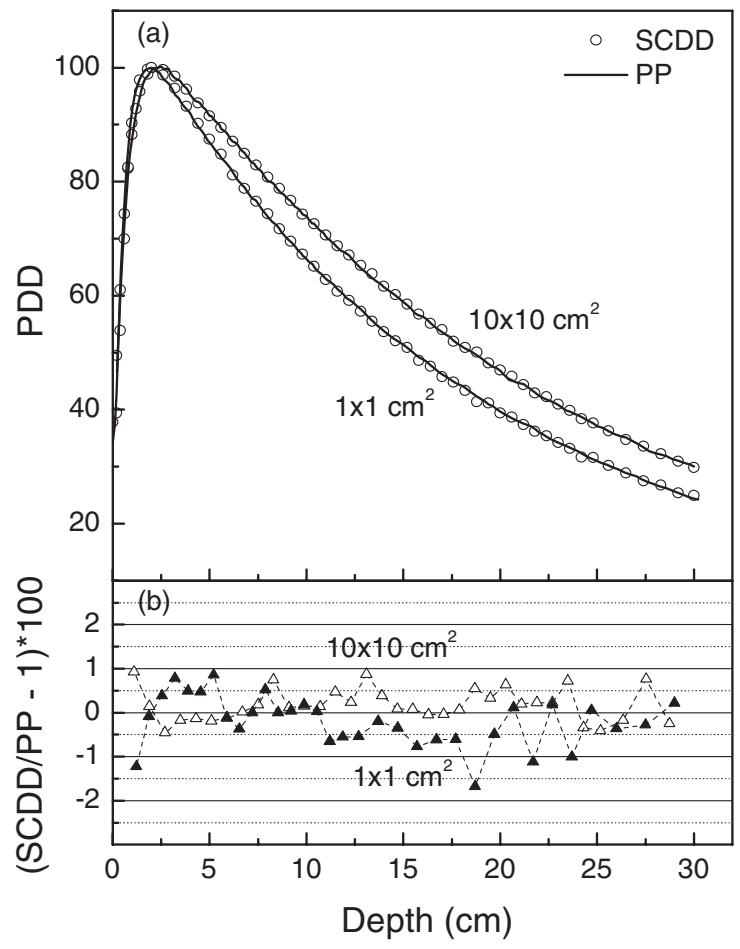

FIG. 5. (a) PDDs measured by the SCDD and the PP-IC in $10 \times 10 \mathrm{~cm}^{2}$ and $1 \times 1 \mathrm{~cm}^{2}$ fields and (b) relative difference between PDDs.

between the profiles measured by two detectors can be observed, thus indicating a very similar spatial resolution of the two devices. Indeed, the active area of the investigated SCDD is defined by the $\sim 2.2 \mathrm{~mm}$ diameter of the Al contact thermally evaporated on the diamond surface, a size which is very similar to that of the PP-IC in vertical orientation. Nonetheless, small deviations with amplitude below 0.04 can be seen in the penumbra region of some difference plots. In these high-gradient regions, such an effect corresponds to differences in the abscissa value (lateral position) of about $0.2 \mathrm{~mm}$. The above experimental results are therefore compatible with the differences between the two devices, e.g., size of the active volume, or small electron transport perturbation due to the different device geometry and materials. ${ }^{11,13}$

More remarkable differences are observed by changing the SCDD orientation with respect to the photon beam axis. The results are summarized in Table II in terms of the $80 \%-$ $20 \%$ penumbra measured by the SCDD and the PP-IC for $1 \times 1 \mathrm{~cm}^{2}$ and $10 \times 10 \mathrm{~cm}^{2}$ square fields, at depths of $2.4 \mathrm{~cm}$

TABLE I. $d_{\max }$ and PDD values at $10 \mathrm{~cm}$ depth as measured by SCDD and PP-IC for all the investigated field sizes, s.

\begin{tabular}{lccccc}
\hline \hline & \multicolumn{2}{c}{ SCDD } & & \multicolumn{2}{c}{ PP-IC } \\
\cline { 1 - 2 } \cline { 5 - 6 } $\mathrm{s}(\mathrm{cm})$ & $\mathrm{d}_{\max }(\mathrm{cm})$ & PDD at $10 \mathrm{~cm}$ & & $\mathrm{~d}_{\max }(\mathrm{cm})$ & PDD at $10 \mathrm{~cm}$ \\
\hline 1 & 1.92 & 0.665 & & 1.94 & 0.670 \\
2 & 2.32 & 0.687 & & 2.30 & 0.690 \\
3 & 2.45 & 0.700 & & 2.43 & 0.701 \\
5 & 2.44 & 0.717 & & 2.45 & 0.715 \\
10 & 2.35 & 0.739 & & 2.38 & 0.737 \\
\hline \hline
\end{tabular}

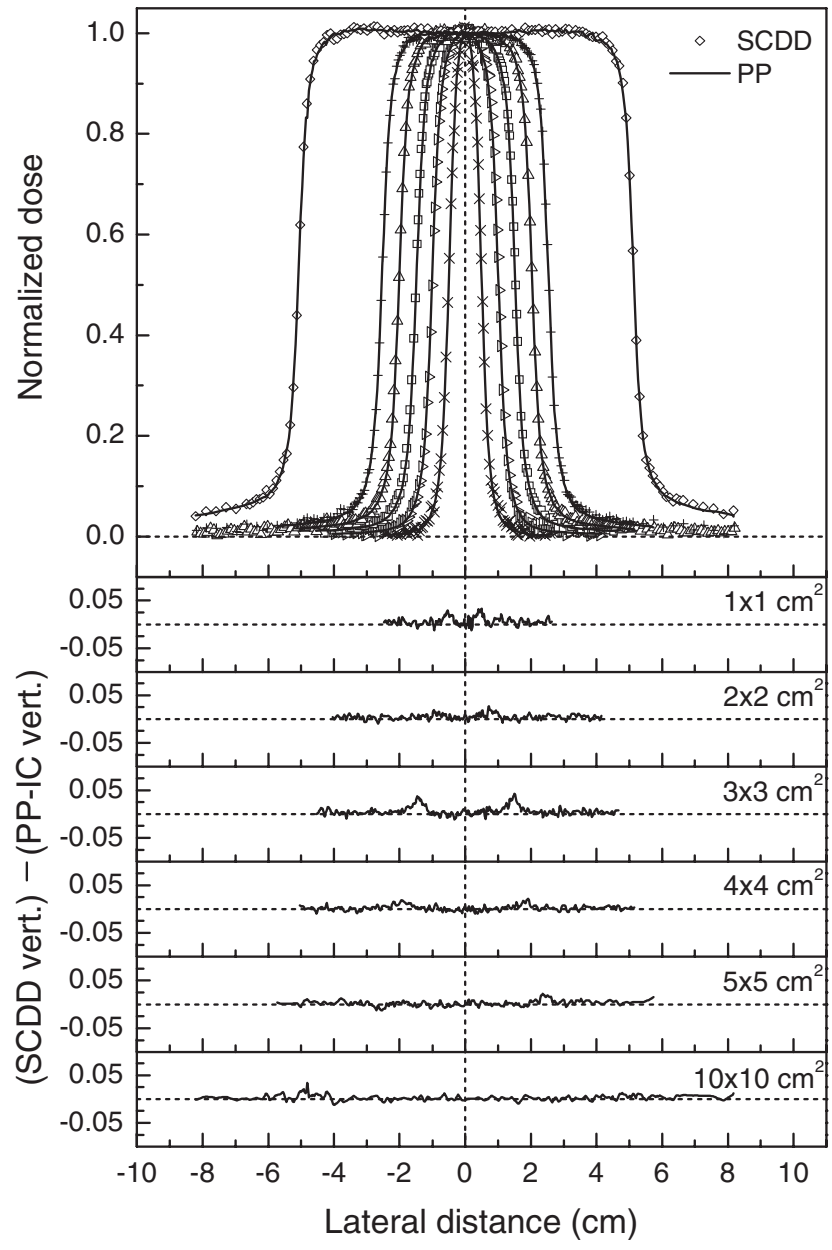

FIG. 6. Normalized in-plane profiles measured at $d_{\max }$ with both the SCDD (symbols) and PP-IC (line) in vertical orientation in several square photon beams. For each examined field size the differences between SCDD and PP-IC profiles are also reported.

and $10 \mathrm{~cm}$, both in vertical orientation and horizontal orientation, with the detector axis parallel to the cross-plane direction in the latter case. In this scan orientation, SCDD is expected to exhibit its highest spatial resolution, since the physical size of the active region along the cross-plane scan axis is only $1 \mu \mathrm{m}$. This is confirmed in Table II and is even more evident in Fig. 7, where the right penumbra region of $2 \times 2$ cross-plane profile and the difference plots are reported. This is done for a more detailed comparison between the SCDD in vertical vs horizontal orientation [Fig. 7(a)] and between the SCDD in horizontal orientation vs the PP-IC in vertical orientation [Fig. 7(b)]. In both cases, the already mentioned penumbra narrowing is clearly visible.

\section{III.D. Output factors}

The output factors normalized at the $10 \times 10 \mathrm{~cm}^{2}$ reference field, obtained for the SCDD and the PP-IC, both positioned in vertical orientation, are shown in Fig. 8(a), together with a relative difference plot [Fig. 8(b)]. A very good agreement can be observed, down to the $1 \times 1 \mathrm{~cm}^{2}$ field size with relative deviation well below $1 \%$ in all the investigated range. 
TABLE II. The $80 \%-20 \%$ in-plane and cross-plane penumbra measured in the $1 \times 1 \mathrm{~cm}^{2}$ and $10 \times 10 \mathrm{~cm}^{2}$ square fields at depths of 2.4 and $10 \mathrm{~cm}$ measured by PP-IC and the SCDD in vertical and horizontal orientation.

\begin{tabular}{|c|c|c|c|c|c|c|c|c|c|}
\hline \multicolumn{6}{|c|}{ SCDD $80 \%-20 \%$ Penumbra $(\mathrm{mm})$} & \multicolumn{4}{|c|}{ PP-IC $80 \%-20 \%$ Penumbra $(\mathrm{mm})$} \\
\hline \multirow[b]{2}{*}{$\operatorname{Depth}(\mathrm{cm})$} & \multirow[b]{2}{*}{ Field size $\left(\mathrm{cm}^{2}\right)$} & \multicolumn{2}{|c|}{ Horizontal } & \multicolumn{2}{|c|}{ Vertical } & \multicolumn{2}{|c|}{ Horizontal } & \multicolumn{2}{|c|}{ Vertical } \\
\hline & & In-plane & Cross-plane & In-plane & Cross-plane & In-plane & Cross-plane & In-plane & Cross-plane \\
\hline \multirow[t]{2}{*}{2.4} & $1 \times 1$ & 3.16 & 2.35 & 3.30 & 3.10 & 3.50 & 4.05 & 3.41 & 3.03 \\
\hline & $10 \times 10$ & 3.94 & 3.81 & 4.90 & 4.72 & 4.86 & 5.56 & 4.88 & 4.30 \\
\hline \multirow[t]{2}{*}{10.0} & $1 \times 1$ & 3.42 & 2.64 & 3.74 & 3.37 & 3.90 & 4.43 & 3.72 & 3.30 \\
\hline & $10 \times 10$ & 4.30 & 4.28 & 5.66 & 5.35 & 6.30 & 6.83 & 6.25 & 5.75 \\
\hline
\end{tabular}

\section{III.E. Angular response}

The angular response of the SCDD was investigated in a $3 \times 3 \mathrm{~cm}^{2}$ field size with respect to the two angles introduced in section II.B: the azimuth angle and the polar angle. The obtained results are shown in Fig. 9 as percentage deviations from the response at 0 degrees, together with a schematic representation of the two orientations studied. As for the azimuth

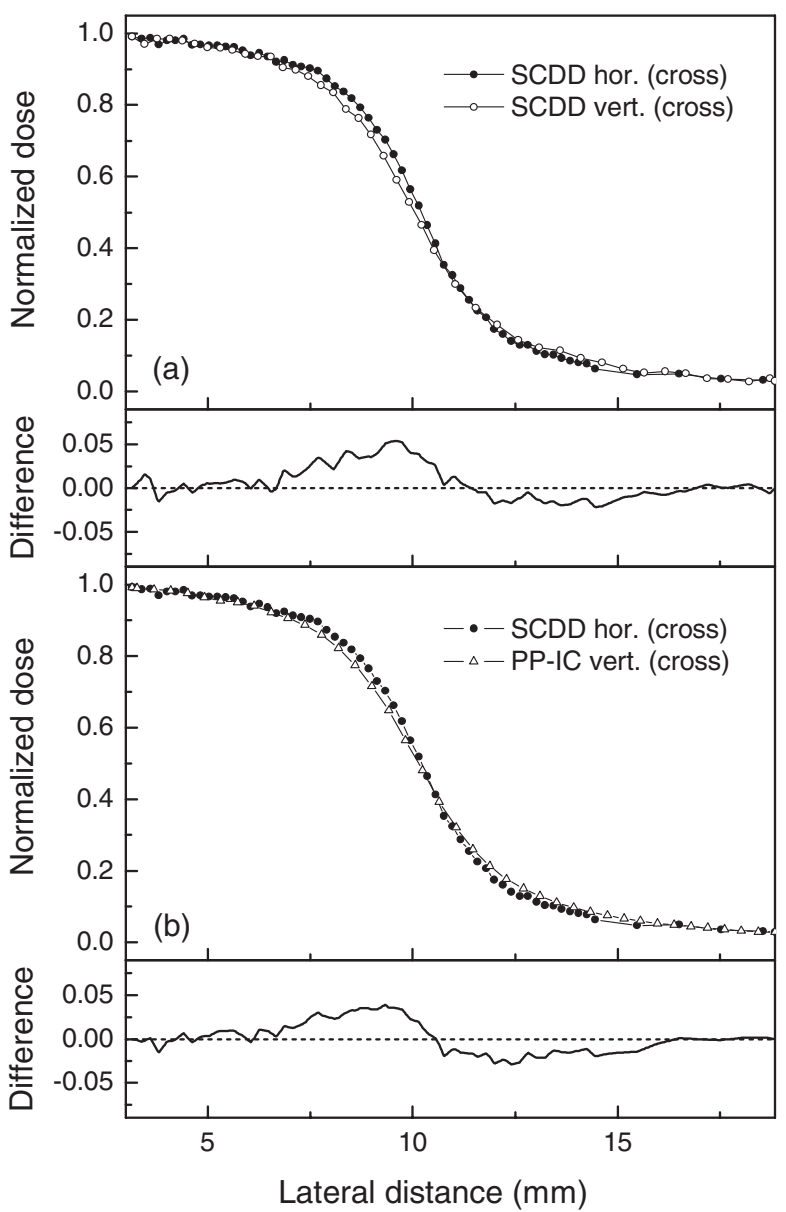

FIG. 7. Right penumbra region of cross-plane profiles measured in a $2 \times 2$ $\mathrm{cm}^{2}$ beam by the SCDD in horizontal orientation compared with SCDD (a) and PP-IC (b) in vertical orientation. The difference plots are also reported. angle, the data reported in Fig. 9(a) show that the response is nearly constant within a $0.5 \%$ fluctuation. The results obtained by varying the polar angle are summarized in Fig. 9(b). In this case the axis of the SCDD was rotated in the range $0^{\circ}-150^{\circ}$ with respect to the irradiation direction. Two different depths were chosen $\left(\mathrm{d}_{\max }\right.$ and $\left.10 \mathrm{~cm}\right)$. The normalized trends almost coincide and a maximum variation in the full angular scale of about $3.5 \%$ can be noticed $\left(1.9 \%\right.$ at $\left.90^{\circ}\right)$. A few measurements were taken in the $-160^{\circ}-0^{\circ}$ range [not reported in Fig. 9(b)], which confirmed a symmetric behavior of the polar angular dependence curve. Although in agreement in the overall trends, such a dependence is somewhat higher than the one reported in literature for PTW natural diamonds. ${ }^{22,41}$ This is possibly due to the intrinsic fabrication asymmetry of our diamond devices, with few $\mu \mathrm{m}$ thick active layer on top of a $\sim 400 \mu \mathrm{m}$ thick substrate. In addition, a significant part of the angular dependence could also arise from the encapsulating materials, their interfaces, the

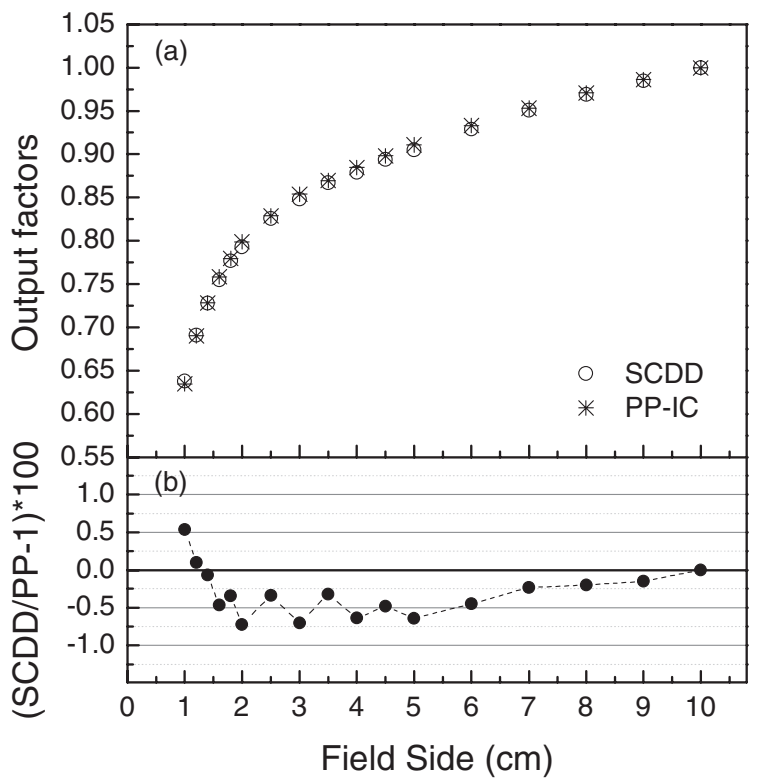

FIG. 8. (a) Output factors measured with the SCDD and the PP-IC for square field sizes varying from $1 \times 1 \mathrm{~cm}^{2}$ to $10 \times 10 \mathrm{~cm}^{2}$ and (b) relative difference between the values from the two detectors. 

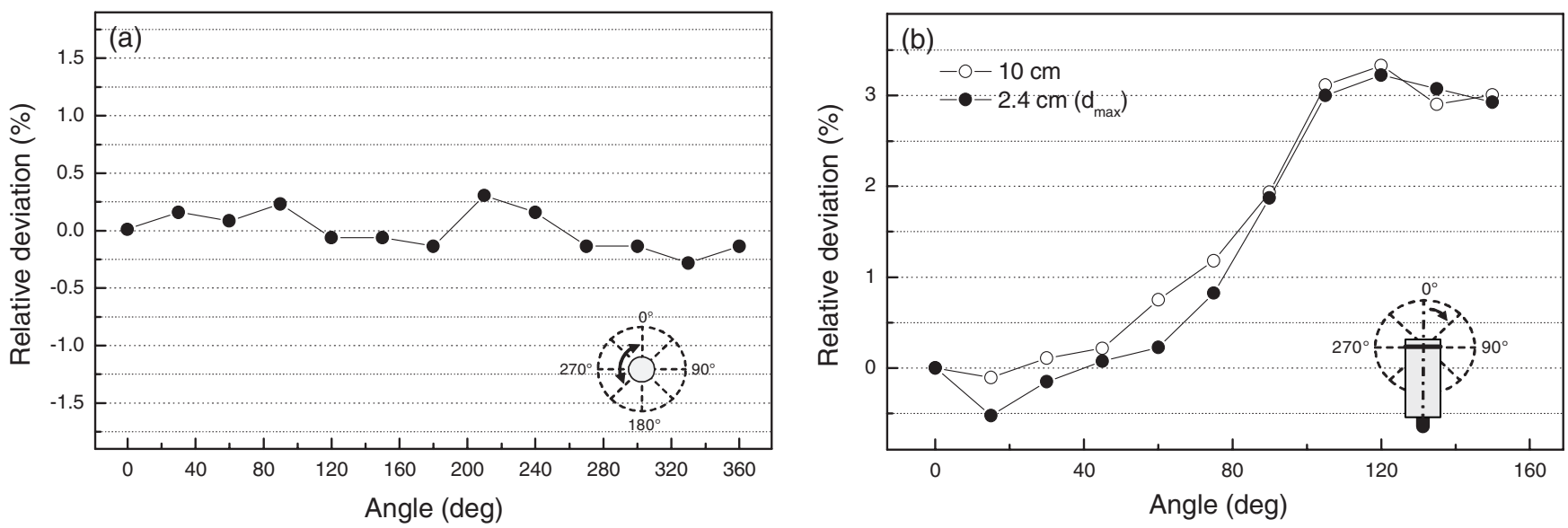

FIG. 9. Angular response of the SCDD in a $10 \mathrm{MV}$ photon beam as normalized deviation from the $0^{\circ}$ value: variations with (a) azimuth and (b) polar angles are shown.

electrode materials, and cables. Minimizing such a dependence is then a challenge also for an improvement of the encapsulation techniques. ${ }^{27}$

\section{III.F. Temperature dependence}

The temperature dependence of the SCDD response was studied in the $18-40^{\circ} \mathrm{C}$ range. Each measurement was repeated three times in order to check the temperature equilibrium and the response stability. The results are shown in Fig. 10 as percentage deviation from the $30{ }^{\circ} \mathrm{C}$ response. A maximum difference of about $\pm 0.2 \%$ was found and a temperature coefficient of $(1.4 \pm 0.2) \times 10^{-4}{ }^{\circ} \mathrm{C}^{-1}$ is derived from the linear best fit of the measured data. Such a low temperature dependence makes the investigated SCDD a suitable device in view of in vivo dosimetry applications.

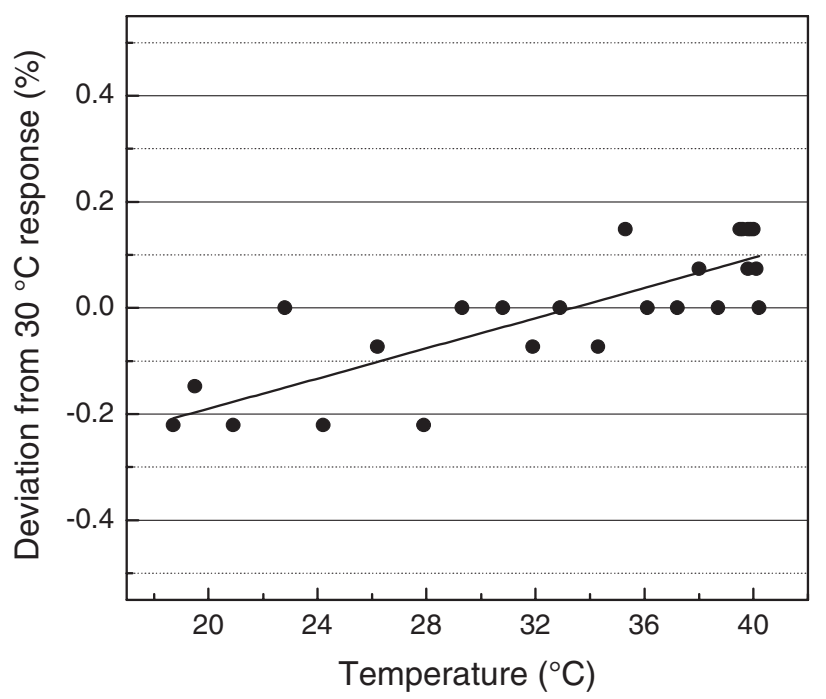

FIG. 10. Temperature dependence of the SCDD response reported as normalized percentage deviation from the $30^{\circ} \mathrm{C}$ value.

\section{SUMMARY AND CONCLUSIONS}

A small volume clinical radiation dosimeter based on a Schottky diode-like CVD synthetic single crystal diamond detector, operated in photovoltaic mode, i.e., at zero bias voltage, was investigated under $10 \mathrm{MV}$ photon beams of different square sizes. Temporal response, including preirradiation dose and response time, dose and dose rate dependence, percentage depth dose curves, beam profiles, output factors, angular response, and temperature dependence were investigated. Particular care was devoted to its characterization in small photon beams, whose dosimetry imposes critical challenges.

A 0.6 Gy preirradiation was found to be necessary in order to stabilize the dosimeter response within $\pm 0.5 \%$. The response time was not directly measured but it was demonstrated to be faster than $0.1 \mathrm{~s}$, corresponding to the $10 \mathrm{~Hz}$ used acquisition rate during the preirradiation sequence.

The dosimetric properties of the proposed diamond detector were assessed by comparing PDDs, beam profiles, and output factors with the ones obtained by a PTW type 31014 PinPoint microionization chamber.

A good linearity of the response as a function of dose was found down to about $1.2 \mathrm{cGy}$, with deviation from linearity within $\pm 0.5 \%$. The dose rate dependence was studied by testing the dosimeter in the $1-6 \mathrm{~Gy} / \mathrm{min}$. Variation below \pm 0.5 was obtained. PDDs and output factors were in good agreement with those measured by the reference ionization chamber with deviations below $1 \%$. Beam profile measurements showed that the diamond dosimeter exhibits a better spatial resolution if placed with its axis perpendicular to the beam direction. In this operating conditions the diamond detector showed a higher resolution with respect to the PinPoint chamber. A negligible angular dependence within $0.5 \%$ from the $0^{\circ}$ response was found as a function of the azimuth angle whereas a more pronounced deviation of $3.5 \%$ was found with the polar angle as expected from the device geometry. The temperature dependence was investigated as well and a 
$\pm 0.2 \%$ deviation from the $30{ }^{\circ} \mathrm{C}$ response was found in the $18-40{ }^{\circ} \mathrm{C}$ range, with a very low temperature coefficient of $(1.4 \pm 0.2) \times 10^{-4}{ }^{\circ} \mathrm{C}^{-1}$.

The reported results indicate the proposed synthetic single crystal diamond Schottky diode as a suitable candidate for application in small radiation fields dosimetry.

\section{ACKNOWLEDGMENTS}

The authors wish to thank "Fondazione Roma" for financial support and G. Luce and S. Morgante for their skilful assistance in the dosimeter fabrication.

a) Author to whom correspondence should be addressed. Electronic mail: gianluca.verona.rinati@uniroma2.it

${ }^{1}$ I. J. Das, G. X. Ding, and A. Ahnesjö, "Small fields: Nonequilibrium radiation dosimetry," Med. Phys. 35, 206-215 (2008).

${ }^{2}$ R. K. Rice, J. L. Hansen, G. K. Svensson, and R. L. Siddeon, "Measurements of dose distributions in small beams of 6 MV x-rays," Phys. Med. Biol. 32, 1087-1099 (1987).

${ }^{3}$ A. Wu, R. D. Zwicker, A. M. Kalend, and Z. Zheng, "Comments on dose measurements for a narrow beam in radiosurgery," Med. Phys. 20, 777-779 (1993).

${ }^{4}$ W. U. Laub and T. Wong, "The volume effect of detectors in the dosimetry of small fields used in IMRT," Med. Phys. 30, 341-347 (2003).

${ }^{5} \mathrm{C}$. McKerracher and D. I. Thwaites, "Assessment of new small-field detectors against standard-field detectors for practical stereotactic beam data acquisition,” Phys. Med. Biol. 44, 2143-2160 (1999).

${ }^{6}$ M. Westermark, J. Arndt, B. Nilsson, and A. Brahme, "Comparative dosimetry in narrow high-energy photon beams," Phys. Med. Biol. 45, 685-702 (2000).

${ }^{7}$ O. A. Sauer and J. Wilbert, "Measurement of output factors for small photon beams," Med. Phys. 34, 1983-1988 (2007).

${ }^{8}$ P. Francescon, S. Cora, and C. Cavedon, "Total scatter factors of small beams: A multidetector and Monte Carlo study," Med. Phys. 35, 504-513 (2008)

${ }^{9}$ C. Martens, C. De Wagter, and W. De Neve, "The value of the PinPoint ion chamber for characterization of small field segments used in intensity modulated radiotherapy," Phys. Med. Biol. 45, 2519-2530 (2000).

${ }^{10}$ S. Agostinelli, S. Garelli, M. Piergentili, and F. Foppiano, "Response to high-energy photons of PTW31014 PinPoint ion chamber with a central aluminium electrode," Med. Phys. 35, 3293-3301 (2008).

${ }^{11}$ E. Pappas, T. G. Maris, F. Zacharopoulou, A. Papadakis, S. Manolopoulos, S. Green, and C. Wojnecki, "Small SRS photon field profile dosimetry performed using a PinPoint air ion chamber, a diamond detector, a novel silicon-diode array (DOSI), and polymer gel dosimetry. Analysis and intercomparison," Med. Phys. 35, 4640-4648 (2008).

${ }^{12}$ M. Stasi, B. Baiotto, G. Barboni, and G. Scielzo, "The behaviour of several microionization chambers in small intensity modulated radiotherapy fields," Med. Phys. 31, 2792-2795 (2004).

${ }^{13}$ A. J. Scott, A. E. Nahum, and J. D. Fenwick, "Using a Monte Carlo model to predict dosimetric properties of small radiotherapy photon fields," Med. Phys. 35, 4671-4684 (2008)

${ }^{14}$ A. S. Beddar, D. J. Mason, and P. F. O’Brien, "Absorbed dose perturbation caused by diodes for small field photon dosimetry," Med. Phys. 21, 1075-1079 (1994).

${ }^{15} \mathrm{C}$. McKerracher and D. I. Thwaites, "Notes on the construction of solid state detectors," Radiother. Oncol. 79, 348-351 (2006).

${ }^{16}$ A. Daşu, P. O. Löfroth, and G. Wickman, "Liquid ionization chamber measurements of dose distributions in small $6 \mathrm{MV}$ photon beams," Phys. Med. Biol. 43, 21-36 (1998).

${ }^{17}$ D. M. Gonzalez-Castano, F. Gomez, L. Brualla, J. V. Rosellò, D. Planes, M. Sancez, and M. Pombar "A liquid-filled ionization chamber for high precision relative dosimetry" Phys. Med. 27, 89-96 (2011).
${ }^{18}$ B. Planskoy, "Evaluation of diamond radiation dosemeters," Phys. Med. Biol. 25, 519-532 (1980).

${ }^{19}$ E. A. Burgemeister, "Dosimetry with a diamond operating as a resistor," Phys. Med. Biol. 26, 269-275 (1981).

${ }^{20}$ M. Heydarian, P. W. Hoban, W. A. Beckham, I. M. Borchardt, and A. H. Beddoe, "Evaluation of a PTW diamond detector for electron beam measurements," Phys. Med. Biol. 38, 1035-1042 (1993).

${ }^{21}$ P. W. Hoban, M. Heydarian, W. A. Beckham, and A. H. Beddoe, "Dose rate dependence of a PTW diamond detector in the dosimetry of a $6 \mathrm{MV}$ photon beam," Phys. Med. Biol. 39, 1219-1229 (1994).

${ }^{22}$ S. N. Rustgi, "Evaluation of the dosimetric characteristics of a diamond detector for photon beam measurements," Med. Phys. 22, 567-570 (1995).

${ }^{23}$ S. N. Rustgi and D. M. Frye, "Dosimetric characterization of radiosurgical beams with a diamond detector," Med. Phys. 22, 2117-2121 (1995).

${ }^{24}$ M. Bucciolini, F. Banci Buonamici, S. Mazzocchi, C. De Angelis, S. Onori, and G. A. P. Cirrone, "Diamond detector versus silicon diode and ion chamber in photon beams of different energy and field size," Med. Phys. 35, 2149-2154 (2003).

${ }^{25}$ C. Manfredotti, F. Fizzotti, A. L. Giudice, G. Mucera, P. Polesello, E. Vittone, Y. A. Mankelevich, and N. V. Suetin, "Growth and characterisation of CVD diamond wires for X-ray detection," Diamond Relat. Mater. 6, 1051-1056 (1997).

${ }^{26}$ A. Fidanzio, L. Azario, R. Kalish, Y. Avigal, G. Conte, P. Ascarelli, and A. Piermattei, "A preliminary dosimetric characterization of chemical vapor deposition diamond detector prototypes in photon and electron radiotherapy beams," Med. Phys. 32, 389-395 (2005).

${ }^{27}$ B. Górka, B. Nilsson, R. Svensson, A. Brahme, P. Ascarelli, D. M. Trucchi, G. Conte, and R. Kalish, "Design and characterization of a tissueequivalent CVD-diamond detector for clinical dosimetry in high-energy photon beams," Phys. Med. 24, 159-168 (2008).

${ }^{28}$ C. Descamps, D. Tromson, N. Tranchant, A. Isambert, A. Bridier, C. De Angelis, S. Onori, M. Bucciolini, and P. Bergonzo, "Clinical studies of optimised single crystal and polycrystalline diamonds for radiotherapy dosimetry," Radiat. Meas. 43, 933-938 (2008).

${ }^{29}$ D. Tromson, C. Descamps, N. Tranchant, P. Bergonzo, M. Nesladek, and A. Isambert, "Investigations of high mobility single crystal chemical vapor deposition diamond for radiotherapy photon beam monitoring," J. Appl. Phys. 103, 054512 (2008).

${ }^{30}$ G. T. Betzel, P. Lansley, F. Baluti, L. Reinish, and J. Meyer, "Operating parameters of CVD diamond detectors for radiation dosimetry," Nucl. Instrum. Methods Phys. Res. A 614, 130-136 (2010).

${ }^{31}$ B. Marczewska, I. Kupriyanov, Yu. N. Pal'yanov, T. Novak, P. Olko, M. Rebisz, and M. P. R. Waligorski, "A study of radiation dosimeters based on synthetic HPHT diamond," Diamond Relat. Mater. 16, 191-195 (2007).

${ }^{32}$ S. Almaviva, M. Marinelli, E. Milani, A. Tucciarone, G. Verona-Rinati, R. Consorti, A. Petrucci, F. De Notaristefani, and I. Ciancaglioni, "Synthetic single crystal diamond diodes for radiotherapy dosimetry," Nucl. Instrum. Methods Phys. Res. A 594, 273-277 (2008).

${ }^{33}$ S. Almaviva, I. Ciancaglioni, R. Consorti, F. De Notaristefani, C. Manfredotti, M. Marinelli, E. Milani, A. Petrucci, G. Prestopino, C. Verona, and G. Verona-Rinati, "Synthetic single crystal diamond dosimeters for Intensity Modulated Radiation Therapy applications," Nucl. Instrum. Methods Phys. Res. A 608, 191-194 (2009).

${ }^{34}$ M. Pimpinella, I. Ciancaglioni, R. Consorti, C. Di Venanzio, A. S. Guerra, A. Petrucci, A. Stravato, and G. Verona-Rinati, "A synthetic diamond detector as transfer dosimeter for $\mathrm{D}_{\mathrm{w}}$ measurements in photon beams with small field sizes," Metrologia 49 (2012) (in press).

${ }^{35}$ S. Almaviva, M. Marinelli, E. Milani, G. Prestopino, A. Tucciarone, C. Verona, G. Verona-Rinati, M. Angelone, M. Pillon, I. Dolbnya, K. Sawhney, and N. Tartoni, "Chemical vapor deposition diamond based multilayered radiation detector: Physical analysis of detection properties," J. Appl. Phys. 107, 014511 (2010).

${ }^{36}$ I. J. Das, C. W. Cheng, R. J. Watts, A. Ahnesjö, J. Gibbons, X. A. Li, J. Lowenstein, R. K. Mitra, W. E. Simon, and T. C. Zhu, "Accelerator beam data commissioning equipment and procedures: Report of the TG-106 of the Therapy Physics Committee of the AAPM," Med. Phys. 35, 4186-4215 (2008). 
37 “Absorbed dose determination in external beam radiotherapy," IAEA TRS No. 398 (IAEA, Vienna, 2000).

${ }^{38}$ J. P. McCaffrey, B. Downton, H. Shen, and M. McEwen, "Pre-irradiation effects on ionization chambers used in radiation therapy," Phys. Med. Biol. 50, N121-N133 (2005).

${ }^{39}$ J. F. Fowler, "Radiation-induced conductivity in the solid state, and some applications," Phys. Med. Biol. 3, 395-410 (1959).
${ }^{40}$ S. F. Kozlov, R. Stuck, M. Hage-Ali, and P. Siffert, "Preparation and characteristics of natural diamond radiation detectors," IEEE Trans. Nucl. Sci. 22, 160-170 (1975).

${ }^{41}$ C. De Angelis, S. Onori, M. Pacillo, G. A. P. Cirrone, G. Cuttone, L. Raffaele, M. Bucciolini, and S. Mazzocchi, "An investigation of the operating characteristics of two PTW diamond detectors in photon and electron beams," Med. Phys. 29, 248-254 (2002). 